\title{
A new data analysis technique in the study of mutual event lightcurves
}

\author{
M. E. Ramírez ${ }^{1}$, Y. Jiménez ${ }^{1}$, M. J. González ${ }^{1}$, M. Berrocoso ${ }^{1}$, and M. Sánchez-Francisco ${ }^{2}$ \\ ${ }^{1}$ Laboratorio de Astronomía y Geodesia, Departamento de Matemáticas, Facultad de Ciencias, Universidad de Cádiz, \\ 11510 Puerto Real, Cádiz, Spain \\ e-mail: mariaeva.ramirez@uca.es \\ ${ }^{2}$ Real Instituto y Observatorio de la Armada en San Fernando, c/Cecilio Pujazón s/n., 11110 San Fernando, Cádiz, Spain
}

Received 9 June 2004 / Accepted 27 October 2005

\section{ABSTRACT}

The purpose of this paper is to develop a new methodology for analyzing astronomical data, in particular, one of the light curves obtained in the PHEMU campaign in 1997, where the mutual phenomena between Jupiter and its satellites were observed from the Earth due to the coincidence of the equatorial plane of Jupiter and the orbit plane of the Earth. The more specific aim of this study is to determine the times of the beginning and the end of the phenomena, and the moment when the recorded light flux was at a minimum.

The techniques we applied were based on the wavelet theory, which allows us to study a signal from its approximations and details at different scales. Before applying the designed algorithm to our experimental data, we considered a synthetic light curve in order to check the reliability of our method. It should be mentioned that the results are not relevant from the astronomical point of view, since no corrections for albedo or illumination were applied to the recorded data.

Key words. celestial mechanics - methods: data analysis - eclipses - planets and satellites: general

\section{Introduction}

In the present paper we show how wavelet theory is a useful tool in analyzing astronomical data, in our case, the study of the light curve obtained from the eclipse of Europe by the satellite Io in the PHEMU campaign during 1997, when mutual phenomena among Jupiter and its Galilean satellites Io (J1), Europe (J2), Ganimedes (J3), and Calisto (J4) were observed.

Every six years, the planes of the orbit of the Earth and of the equator of Jupiter coincide, so it is possible to observe from the Earth the interactions taking place among this planet and these satellites. These phenomena can be divided into two groups: those where Jupiter and one of its satellites interact, called classical events, and those that take place between two of the Galilean satellites, called mutual phenomena. The lack of atmosphere over these satellites means that data are free of corrections because of refraction and dispersion.

The importance of studying the interactions among Jupiter and its Galilean satellites lies in the fact that this system behaves like the Solar System. Thus, knowing its dynamics helps us to understand ours better. The observation campaigns organized from 1985 for this purpose are called PHEMU campaigns, and observatories all over the world take part in order to increase the amount of data available for analysis (Arlot 1987).

Our purpose in this paper is to develop a new methodology for analyzing the data obtained from these events, in particular, estimating the time of beginning and end of the phenomena, along with the moment were the registered light flux reaches its minimum. We will only concentrate on the mathematical aspect of the data analysis. Instead of the usual polynomial adjustment, we propose a method that first consists in removing the noise from the signal, and then in determining those points we are interested in by noticing that they correspond to sudden changes in the signal behavior. It turns out that the wavelet basis provides the correct setting for carrying out this program.

To denoise the signal we applied the coherent structure technique (Mallat 1999) to the wavelet coefficients, which consists of preserving those that satisfy certain conditions and cancelling the others. The signal is then recovered using these new filtered coefficients. To estimate the required times, we designed an algorithm based on the fact that modulus maxima wavelet coefficients determine both the isolated singularities and the sudden changes in a signal. The proposed algorithm was first applied to a synthetic curve in order to check its reliability.

The first three sections of this paper deal with the description of the campaign: some historical background, its objectives, and the equipment used to observe the phenomena.

In the fourth section we detail the way these observations were carried out. The magnitude to observe is the light flux received from the sky, which corresponds to the luminosity of 
the sky background plus the luminosity of the interacting satellites. It allows us to determine the times where the event begins, when it ends, and the moment when the recorded luminosity is at its minimum.

The fifth section is about the processing of the data by means of the wavelet theory. We introduce its basic aspects and the new point of view it provides, since it allows a signal to be studied at different scales. The methodology for analyzing these data is also treated in this section, where wavelets are applied with two different purposes: to denoise the signal and to determine the times we are interested in. At the end of the section it is shown how this procedure works in studying of the eclipse of Europe by the satellite Io and results obtained by using a different wavelet basis are presented. A final discussion follows in the last section.

\section{PHEMU campaigns and their objectives}

Due to tie effects and volcanic eruptions, among other factors, the movement of Jupiter's satellites constitutes one of the most difficult problems in celestial mechanics. Measurements during mutual events let us determine the spatial position of the satellites with an accuracy of $100 \mathrm{~km}$, which can sometimes decrease down to $30 \mathrm{~km}$.

The study of these phenomena began in 1979, but in 1985 the Bureau des longitudes in Paris designed a network of observatories all over the world with the aim of increasing the amount of collected data (Arlot et al. 1997). A detailed description of the observations, as well as the results, can be found in Arlot et al. (1992). The Royal Naval Observatory at San Fernando (ROA) took part for the first time in 1997. The members of the campaign were some observers from the ROA, from the Mathematics Department of the University of Cádiz, and from the Astronomy Association in San Fernando.

The goal of these campaigns is to understand the dynamics of the Jovian System from the observation of the events at different observatories in the world, by detecting the initial time, the end time and the time of maximum darkness in the phenomena.

The classical and the mutual phenomena that can occur are the following: eclipses, where a satellite immerses in the shadow of Jupiter or another satellite; occultations, when a satellite disappears and appears behind another one or behind the planet; and transits, when a satellite passes in front of Jupiter or another satellite. Both classical or mutual phenomena can be annular, partial, or total (Figs. 1 and 2; Arlot 1997).

\section{Equipment}

Observations were carried out using a Gauthier Astrograph set up in the ROA and consisting of a double refractor telescope: with a visual telescope, with a lens of $20 \mathrm{~cm}$ aperture and $360 \mathrm{~cm}$ focal distance, and a photographic telescope of two lenses of $33 \mathrm{~cm}$ aperture and $346 \mathrm{~cm}$ focal distance.

An RCA-931 photomultiplier was connected to the analogical-digital card of a computer and put into operation on the telescope focal plane to register the changes produced in the light energy of the observed events. One $\mathrm{mm}$ matches

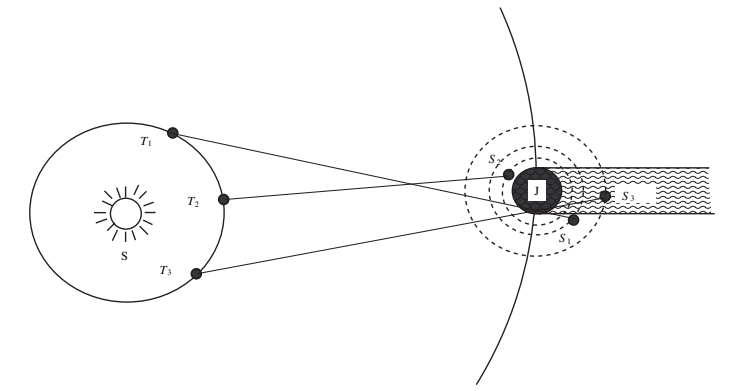

Fig. 1. Geometric configuration: for an observer at $T_{1}$, the satellite $S_{1}$ is occulted by Jupiter; for an observer at $T_{2}$, satellite $S_{2}$ appears to move across the face of Jupiter, which is defined as a transit; at $T_{3}$, satellite $S_{3}$ is eclipsed by the cone shadow of Jupiter.

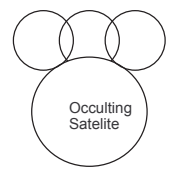

(a)

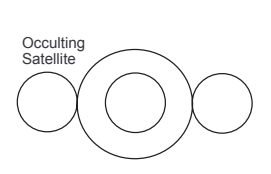

(b)

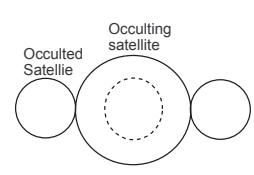

(c)
Fig. 2. Example of: a) partial occultation; b) annular occultation; c) total occultation.

in the focal plane with $1^{\prime \prime}$ of the celestial sphere. A link to the UTC time scale from the Time Department in the ROA was available to time the records. Due to the astrograph dome, the minimum altitude for observing Jupiter was $20 \mathrm{deg}$.

The width of the diaphragm depends on the configuration of the satellites interacting; too wide a diaphragm will let brightness from other satellites or even from Jupiter pass through, thereby contaminating the signal and influencing the results. The diaphragm used for the observations carried out in San Fernando takes in 59" of sky. As a reference point, we can point out that the observation of a mutual phenomena, using photoelectric photometry and a diaphragm of $40 \mathrm{~cm}$ of wideness, involves an estimated error of $48 \mathrm{~km}$. No filter was used in the first stage. A preliminary analysis just consisted of plotting the light flux registered versus the integrating time. The reliability of the data was checked straight away.

\section{Observation of the events}

The registered flux light is represented in a plot similar to Fig. 3. Before the event starts and after it ends, the total flux is the sum of the eclipsed or occulted satellite, the eclipsing or occulting one, the flux corresponding to the sky background, and the dark current of the telescope.

Once the event has begun, the light decreases until it reaches a minimum value. In the occultations, both satellites blend in a light point that loses part of its brightness until it reaches the minimum value, whereas in the eclipses, we have just one satellite in the visual field whose brightness drops by an amount that depends on the kind of the event (annular, partial or total). After this point, the light flux increases, and it comes to its initial value at the end of the event, when the interacting satellites move away from each other.

The perfect situation is a curve like the one in Fig. 3, which is symmetrical with respect to its minimum value and constant 


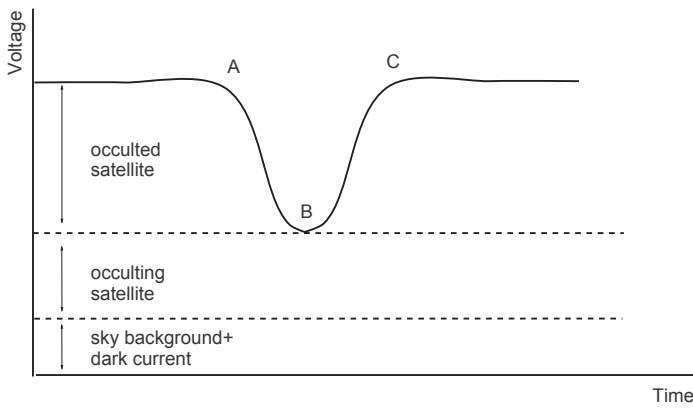

Fig. 3. Components of the registered light flux for a total occultation. Time A matches the beginning of the event, time B the moment of the minimum flux, and time $\mathrm{C}$ the end of the phenomena. For the case of an eclipse we get a similar curve.

before the phenomenon begins. But several factors like the relative movements of the satellites, the changes in the sky background, and the appearance of stars in the visual field make data move away from this ideal situation. In practice, we get a cloud of points that is quite different from the theoretical curve, as shown in Fig. 4.

The sky background and the interacting satellites are registered before and after the event, as well as several times during the observation, especially if it occurs at dusk. A reference object is taken into account for calibration: in a mutual eclipse, this is the eclipsing satellite, since it is present in the visual field during the whole event; in the occultations, if the eclipsing satellite is too close or too far from the eclipsed one, both satellites are observed individually before and after the event, and during the occultation a third satellite is put in the field as the reference object.

If this configuration is not possible, we proceeded as follows: for short events lasting less than $15 \mathrm{~min}$, the reference object is observed before and after the phenomenon, while for long phenomena, the telescope is pointed at it every 5 or $10 \mathrm{~min}$ (Arlot 1997). Due to the relative movements of the satellites, the velocity of monitoring is fixed every night.

Records must be made in advance because events occur a few seconds before and also some minutes after they are predicted. In practice this involves differences of many hundreds or several thousands of $\mathrm{km}$ in the position of the satellites.

It is advisable that each point in the curve be timed with better accuracy than $0.5 \mathrm{~s}$, and the integrating time should not be higher than 1 or $2 \mathrm{~s}$. In our case, we got a record every $0.84 \mathrm{~s}$ approximately.

According to the predictions, 44 mutual phenomena should have been visible from San Fernando, but only 24 of them were recorded due to factors such as bad weather conditions or failures in the equipment or observers' errors. Just six of the 24 were kept since they were the only ones that represented an event clearly: the eclipse of Europe by Io, eclipse of Ganimedes by Europe, the occultation of Io by Ganimedes, the partial occultation of Europe by Ganimedes, and the total occultation of Europe by Ganimedes (Fig. 4).

It is observed that events (d) and (f) could not be completed, since the presence of clouds made the register of the whole phenomenon imposible. Otherwise plots (b), (c), and (e) are similar to the expected curve. Thus we will concentrate only on the eclipse of Europe by Io, in (a).

\section{Data processing}

As stated before, the main purpose of our study is to detect the times that match the beginning of the event $t_{\text {ini }}$, the moment of minimum light flux $t_{\mathrm{min}}$, and the end of the event $t_{\mathrm{end}}$.

In order to do so, we first eliminate the noise in the data by means of the coherent structure procedure, which will be explained in what follows. Then we search for the maxima curves, to detect the sudden changes in the signal, which should correspond to the three instants of time we are trying to estimate. Both techniques are based on the wavelet theory, and the effectiveness of the method is proved by applying our algorithm to a synthetic light curve designed for this purpose. Let us first begin by briefly introducing some background and later on we will analyze the steps of the filtering deeply and search for maxima curves.

\subsection{Wavelet background}

Through over the years, Fourier analysis has been the main tool for signal processing. The Fourier transform is reversible in the sense, that under certain conditions, it lets us move from the study in the time domain to one in the frequency domain, although studying of both domains simultaneously is not possible, as the Heisenberg principle asserts. In fact, the Fourier transform informs about the frequencial components in the signal, but does not show when they occur.

The basis of the wavelet theory is the existence of two functions $\varphi$ and $\psi$, known as father and mother wavelet, respectively. By the operations of translation and dilation, we can, on one hand, analyze the time domain since we translate the functions $\varphi$ and $\psi$ in time, and on the other hand, the dilation or contraction of their time support implies the contraction or dilation of its frequencial support, respectively, which allows us to perform a study in the frequency domain. The importance of these functions comes from the possibility of obtaining a basis of the space of functions of finite energy $L^{2}(\mathbb{R})$. Thus, any function $f \in L^{2}(\mathbb{R})$ can be written in terms of the system $\left\{\psi_{j, k}\right\}_{(j, k)}$ as follows:

$f=\sum_{j \in \mathbb{Z}} \sum_{k \in \mathbb{Z}}\left\langle f, \psi_{j, k}\right\rangle \psi_{j, k}$,

where

$\psi_{j, k}(t)=\frac{1}{2^{j / 2}} \psi\left(\frac{t-2^{j} k}{2^{j}}\right)$.

The coefficients $d_{j, k}=\left\langle f, \psi_{j, k}\right\rangle$ are called wavelet coefficients and are obtained by the inner product of the signal $f$ with the wavelet function $\psi$ localized in time and frequency, that is,

$d_{j, k}=\left\langle f, \psi_{j, k}\right\rangle=\int_{-\infty}^{+\infty} f(t) \frac{1}{2^{j / 2}} \psi\left(\frac{t-2^{j} k}{2^{j}}\right) \mathrm{d} t=f \star \psi_{j}[k]$,

where $f \star \psi_{j}$ denotes the convolution of the signal $f$ by the scaled version $\psi_{j}$ of $\psi, \psi_{j}(t)=2^{-j / 2} \psi\left(t / 2^{j}\right)$. 


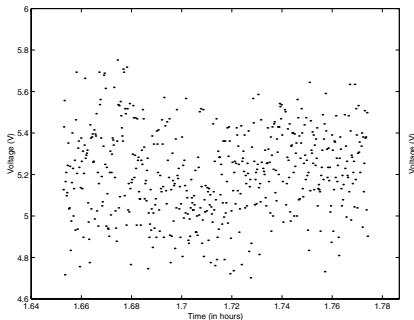

(a)

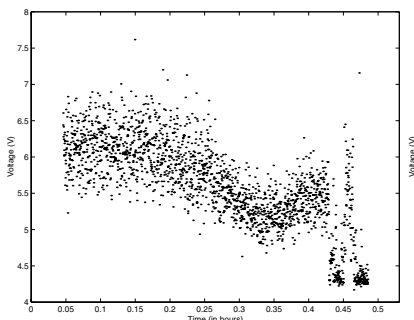

(d)

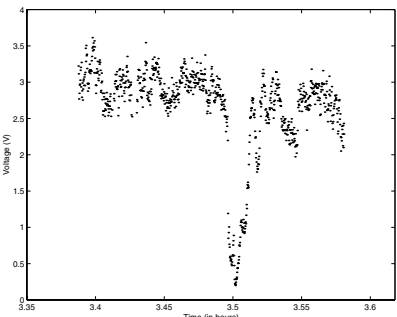

(b)

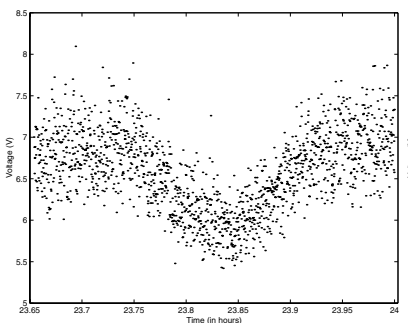

(e)

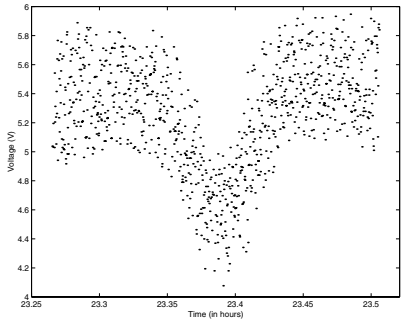

(c)

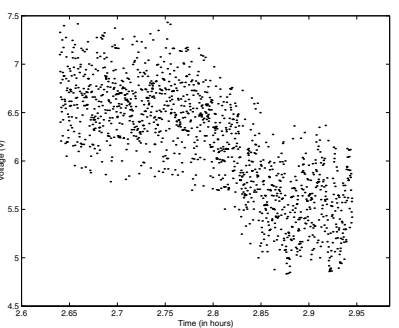

(f)

Fig. 4. Events observed at the Real Instituto y Observatorio de la Armada in San Fernando (Cádiz) during the PHEMU97 campaign. Time is plotted against light flux: a) J1ECLJ2 (07.07.97); b) J2ECLJ3A (16.07.97); c) J3OCC J1P (26.07.97); d) J4ECLJ3A (01.08.97); e) J3OCCJ2P (25.07.97); f) J3OCC J2T (02.08.97). J1, J2, J3, and J4 denote the Galilean satellites from the closest to Jupiter.

The inner product of the signal $f$ with the dilated and translated function $\varphi$, gives

$a_{j, k}=\left\langle f, \varphi_{j, k}\right\rangle=\int_{-\infty}^{+\infty} f(t) \frac{1}{2^{j / 2}} \varphi\left(\frac{t-2^{j} k}{2^{j}}\right) \mathrm{d} t=f \star \varphi_{j}[k]$.

The difference between both expressions lies in the fact that coefficients $a_{j, k}$ provide approximations or averages of the $2^{j}$-scaled signal, while coefficients $d_{j, k}$ determine its details at the same scale.

Under these considerations, each $2^{j}$-scaled approximation $a_{j}$ of a signal can be understood as the sum of the approximation of it at a coarser level $2^{j+1}$, plus the details that appear at level $2^{j}$ and disappear at level $2^{j+1}$, that is,

$a_{j}=a_{j+1}+d_{j+1}$.

The revolution of wavelet theory comes precisely from this fact: with the wavelet transform we can "look at" our signal at different levels. In this way, not only is the study of the signal possible in the time domain, but so is studying it in the frequency domain by changing the scales.

We must notice that the higher the considered scale, the bigger the time support of the function $\psi_{j}$, and the smaller its frequency support becomes. This fact is known as the Heisenberg uncertainty principle (Vetterli \& Kovacevick 1995), which states the impossibility of knowing the exact time and frequency representations of a signal simultaneously. Therefore, we can make the time support of the $\psi_{j}{ }^{\prime} s$ functions decrease and localize our signal in this domain, which is particularly useful for detecting singularities or for making their frequency support decrease, and for localizing the signal in the frequency domain, which is very interesting for denoising, since it allows us to discover the high frequencies in the signal that are usually related to noise.
Related to the size of the time support of a wavelet function is the number of vanishing moments it has. A function $\psi$ is said to have $m$ vanishing moments if it verifies

$\int_{-\infty}^{+\infty} t^{n} \psi(t) \mathrm{d} t=0, \quad$ for $0 \leq n \leq m-1$.

The importance of these vanishing moments comes from the fact that for a function $f$ that is regular enough, which can be written as a power series, each wavelet coefficient $d_{j, k}=$ $\left\langle f, \psi_{j, k}\right\rangle$ will depend only on the terms of order higher than $m$. Thus, function $f$ can be represented from a few non-zero wavelet coefficients (Vetterli \& Kovacevick 1995), which concentrate its energy. This property will be essential in the denoising techniques we apply later on.

On the other hand, if $\psi$ has $m$ vanishing moments, the size of its support will be greater than or equal to $2 m-1$ (Hwang $\&$ Mallat 1991). Thus, in practice, when it comes to the choice of the mother wavelet, we need both a wide support to denoise and a small support to detect singularities or sudden changes in the signal. An arrangement between denoising and detection must be reached.

\subsection{Denoising}

The denoising procedure is based on a wavelet thresholding method; that is, the wavelet coefficients above a certain threshold are preserved and the rest are set to zero. Mallat (1999) pointed out several techniques of non-linear filtering to remove different kinds of noises. Donoho \& Johnstone (1994) proved that, in the case of Gaussian white noise, nearly optimal estimators are obtained by thresholding decomposition coefficients in an orthonormal wavelet basis. 
According to it, the value of the threshold $T$ for estimating a signal in a Gaussian white noise of variance $\sigma^{2}$ should be

$T=\sigma \sqrt{2 \log N}$

where $N$ is the sample size of the noisy data.

Without any a priori knowledge of the type of noise, it is more convenient to use a method that does not involve the noise variance. Mallat (1999) proposes the coherent structure method in this case. The general idea is that noise is defined as the signal components that do not have any strong correlation with the elements in the wavelet basis, that is, the ones below the corresponding threshold. The signal is then estimated by isolating the components that carry the information, i.e., the ones with high correlation, the coherent structures.

According to this, the best basis for denoising will be the one that concentrates the signal energy better over a few coefficients, that is, the one that is capable of distinguishing the coherent structures from the noise.

Following the coherent structures algorithm, the wavelet coefficients $d_{j, k}$ (Mallat 1999) are calculated and arranged by modulus in the following way

$\left|\alpha_{1}\right| \geq\left|\alpha_{2}\right| \geq \ldots \geq\left|\alpha_{N}\right|$

where $N$ represents the sample size and $\alpha_{i}$ denotes the detail coefficient $d_{j, k}$ in the proper position according to Eq. (8). In such a way, signal $f$ can be written as follows:

$f=\sum_{i=1}^{N} \alpha_{i} \psi_{i}$

where $\psi_{i}$ is the translated and scaled version of the wavelet function $\psi$ corresponding to the wavelet coefficient $\alpha_{i}$.

For each $k \geq 0$, the wavelet coefficient $\alpha_{k}$ is not reduced to noise if

$\frac{\left|\alpha_{k}\right|^{2}}{\|f\|^{2}}>T_{N}^{2}=\frac{2 \log N}{N}$,

and $\psi_{k}$ is a coherent structure if

$$
\frac{\left|\alpha_{i}\right|^{2}}{\sum_{i=k}^{N}\left|\left\langle f, \psi_{i}\right\rangle\right|^{2}}=\frac{\left|\alpha_{i}\right|^{2}}{\sum_{i=k}^{N}\left|\alpha_{i}\right|^{2}}>T_{N-k+1}^{2} \text {. }
$$

The algorithm stops when we find the first index $k=M$, such that $\psi_{M}$ is not a coherent structure. Filtered coefficients will be given by

$\tilde{\alpha}_{i}=\left\{\begin{array}{ll}\alpha_{i}, & i<M \\ 0, & i \geq M\end{array}\right.$.

Then, signal $\tilde{f}$ is estimated by the sum of the $M$ coherent structures:

$\tilde{f}=\sum_{i=1}^{M-1} \alpha_{i} \psi_{i}$.

The advantage of this procedure over a filtering by frequency bands lies in the fact that we get a signal that is almost free of noise by hardly modifying the characteristics of the signal, while in traditional filtering the denoising involves a smoothness of the original components of the signal. In fact, a wavelet thresholding is equivalent to estimating the signal with a smoothing that is locally adapted to the signal regularity.

\subsection{Maxima curves}

Irregular structures of a signal (discontinuities, inflection points, local maxima or minima, sudden changes, etc.) usually offer important information about it. From a continuous point of view, the wavelet transform can be used to study the local regularity of a signal. We have mentioned that one of the main advantages of the wavelet theory lies in how it allows the analysis of the local structures with a zooming procedure that reduces the scale parameter. When the scale decreases, the wavelet transform measures the fine scale variations in the neighborhood of certain point. Thus, a fast decay of the wavelet transform as the scales go to zero implies that the signal is regular at that point. Several results relating the decay of the wavelet transform to the local regularity of a signal can be found in Hwang \& Mallat (1991).

The designed algorithm is based on the following result: modulus maxima wavelet (MMW) coefficients determine the irregular structures in the signal, which appear in every scaled version. Considering these modulus maxima along the successive scales, a line called maxima curve is defined. Thus, singularities are detected by finding the abscissa where this maxima curve converges at fine scales (Hwang \& Mallat 1991). In order to determine such abscissa with good precision, we need a wavelet with small time support.

The number of vanishing moments of the wavelet function $\psi$ is also related to the regularity of a signal, since they determine the "kind of singularity" we will be able to detect. We can gather from the theoretical aspect of the light curve that the three points we want to detect are those with the maximum curvature.

It can be proved (Mallat 1999) that a wavelet function $\psi$ with a fast decay has $n$ vanishing moments, if and only if $\psi$ is the nth derivative of a function $\theta$ with fast decay, that is,

$\psi(t)=(-1)^{n} \frac{\mathrm{d}^{n} \theta(t)}{\mathrm{d} t^{n}}$

Thus, if a wavelet $\psi$ has just one vanishing moment, the wavelet transform of a signal $f$ at a scale $2^{j}$ can be written as

$$
\begin{aligned}
f \star \psi_{j}(t) & =f \star\left(2^{-j / 2} \psi\left(\frac{t}{2^{j}}\right)\right)=f \star\left(-2^{-j / 2} \theta^{\prime}\left(\frac{t}{2^{j}}\right)\right) \\
& =f \star 2^{j} \bar{\theta}_{j}{ }^{\prime}(t)=2^{j}\left(f \star \bar{\theta}_{j}\right)^{\prime}(t),
\end{aligned}
$$

where $\bar{\theta}_{j}=-2^{j / 2} \theta\left(t / 2^{j}\right)$. That means that the local maxima of the wavelet transform $f \star \psi_{j}$ at scale $2^{j}$ are the maxima of the first order derivative of the signal $f$ smoothed by a function $\bar{\theta}_{j}$, that is, the inflection points. In a similar way, if the wavelet function $\psi$ has two vanishing moments, it can be proved that the modulus maxima of the wavelet transform

$$
f \star \psi_{j}=\left(2^{j}\right)^{2}\left(f \star \bar{\theta}_{j}\right)^{\prime \prime}(t)
$$

correspond to locally maximum curvatures of $f \star \theta_{j}$.

Therefore, since the three instants of time we want to detect are those matching the highest curvatures and inflection points, we choose wavelet functions with at least two vanishing moments. 


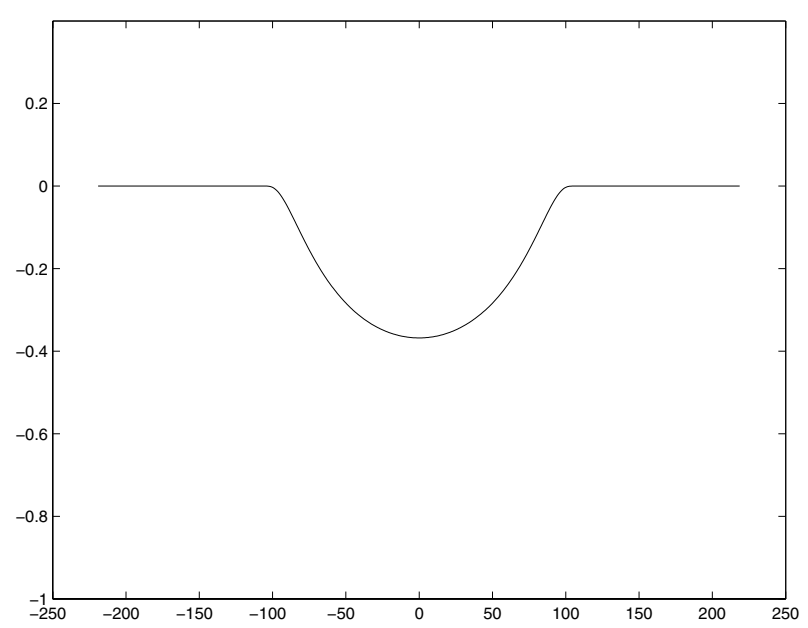

Fig. 5. Simulated light curve.

\subsection{Methodology}

In order to check the reliability of our method, the algorithm was applied to a synthetic light curve (Fig. 5) given by a normalized and discretized version of the following function

$f(x)=\left\{\begin{array}{ll}0, & |x|>1 \\ -\mathrm{e}^{-1 /\left(1-x^{2}\right)}, & |x|<1\end{array}\right.$.

The continuous curve is a differentiable function with derivatives of all orders. The three instants of time are calculated analytically by evaluating the minimum of the curve and those points where the curvature reaches its maxima, thereby obtaining:

$t_{\text {ini }}^{a}=-98.46, t_{\text {min }}^{a}=0, t_{\text {end }}^{a}=98.46$.

The sample size $N=520$ and the sampling rate $0.84 \mathrm{~s}$ are the same as in the registered event, in order to get a simulated curve that is as similar to the experimental situation as possible. We associated the times in Eq. (18) to the closest ones in the synthetic light curve, that is,

$t_{\text {ini }}^{s}=-98.88, t_{\min }^{s}=0.24, t_{\text {end }}^{s}=98.52$.

White noise with different levels of $S N R$ ranging from 1 to $40 \mathrm{~dB}$ was added to the synthetic signal and the method was applied to the noisy data with the aim of getting the results given in Eq. (19). Figures 7a and c represents the noisy and the denoised light curves corresponding to $S N R=30 \mathrm{~dB}$ and $S N R=1 \mathrm{~dB}$, respectively.

Data were filtered using the procedure explained in Sect. 5.2. The wavelet basis is chosen according to the minimum entropy criterion: the best will be the one that better concentrates the signal energy over a few coefficients. In our case, we get different wavelet bases depending on the SNR of the noisy signal. For example, for the signal in Fig. 7a, with a $S N R$ of $30 \mathrm{~dB}$, the most suitable function is the Meyer discrete wavelet basis. On the other hand, for the signal in Fig. 7c with an $S N R$ of $1 \mathrm{~dB}$, the most suitable wavelet is the Coiflet basis with 6 vanishing moments.

To estimate the times given in Eq. (19) we designed an algorithm to detect the maxima curves. First, wavelet

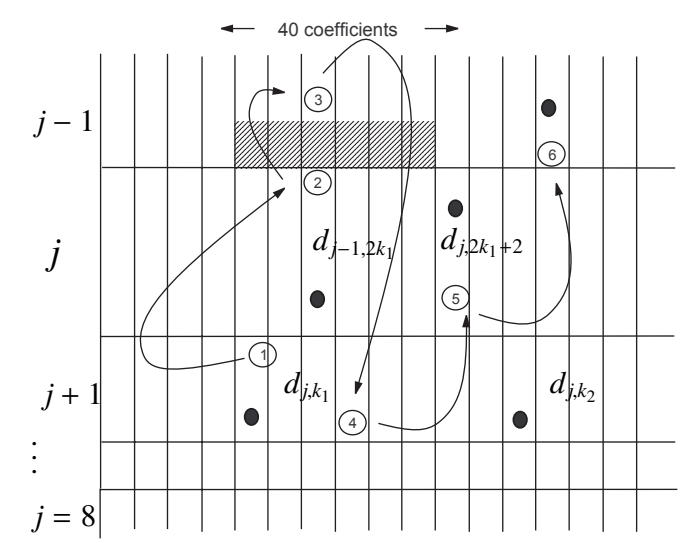

Fig. 6. Iterative algorithm scheme: (1) modulus maxima wavelet coefficients (MMW) are marked at each level and a first one is chosen at the coarser level $j+1$. To select the MMW at level $j$, we search in the forty cells above; (2) if there is more than one, we take the one with greatest modulus, and continue at the level $j-1$; (3) if there is not any MMW, we go down to level $j+1$ (4); and run the algorithm again, choosing the subsequent MMW at level $j(5)$.

coefficients $\left\{d_{j, k}\right\}$ at scales $j=1, \ldots, 8$ were calculated for the filtered signal according to the filter bank algorithm proposed by Mallat (1999). At scale $j$ the number of wavelet coefficients will be $N / 2^{j}$, where $N$ is the sample size. In order to get the same number of coefficients at every scale, wavelets coefficients $\left\{d_{j, k}\right\}_{j=1, \ldots, 8}$ were upsampled and synthesized by means of the reconstruction filters in the algorithm. Therefore, at each scale $j$ we have a component of the original signal of size $N$, and we can find the local modulus maxima wavelet coefficient at each scale $j$ (Figs. 7b and d) that we will denoted by MMW coefficient.

To determine the maxima curves we set the following criterium: an MMW coefficient at scale $j-1$ is said to lie on the same maxima curve as an MMW coefficient at scale $j$, if the first one is located in one of the forty positions above the second one. If there is more than one modulus maxima point at these positions, we take the largest one (Fig. 6). If there is not any, we return to the scale $j+1$ and run the algorithm again. Some packets from the free software WaveLab, available on-line in the URL http://www-stat.stanford.edu./ ${ }^{\sim}$ wavelab, have been used in the design of the algorithm.

To decide which wavelet fits better for detection, we must take into account the existing relation between the number of vanishing moments and the different kinds of singularities, or irregular structures in a signal. In fact, at least two vanishing moments and a small time support are required to detect highest curvatures and inflection points. We ran the algorithm with several wavelet functions satisfying these considerations. Those that give rise to a more clearly defined maximum curve are the Coiflet function with two vanishing moments (coif1) and the Biorthogonal function with two vanishing moments (bior2.2). They both detect three maxima curves, which begin with MMW coefficients from the coarsest level $j=8$ and end at a finer scale $j=2$.

Table 1 summarizes the results $\left(t_{\mathrm{ini}}, t_{\mathrm{min}}, t_{\mathrm{end}}\right)$ obtained after applying our algorithm using a Biorthogonal basis to the simulated light curve. It can be observed that we obtain very 


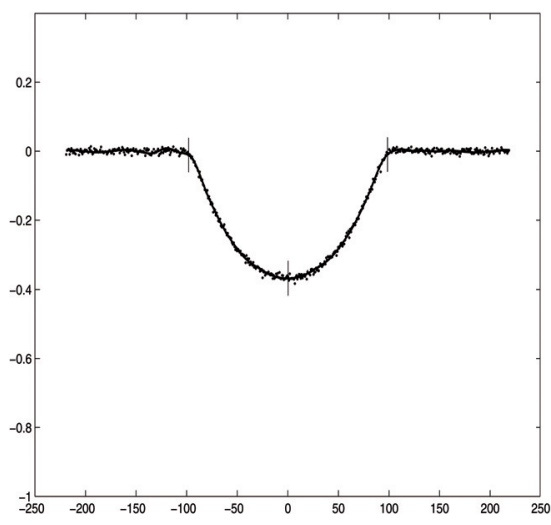

(a)

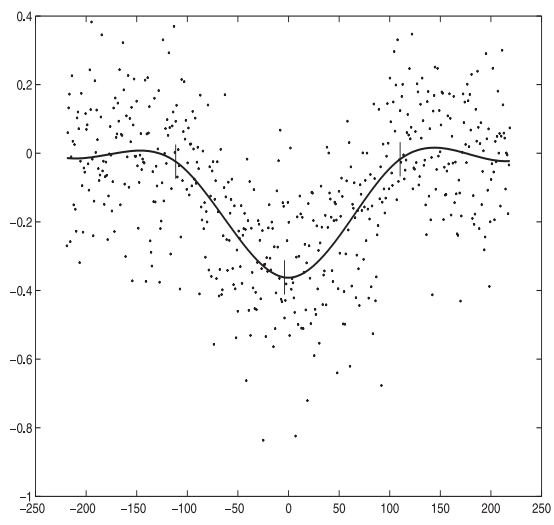

(c)

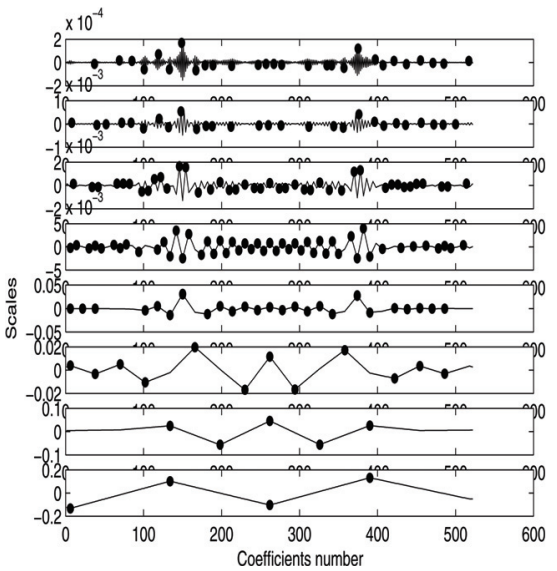

(b)

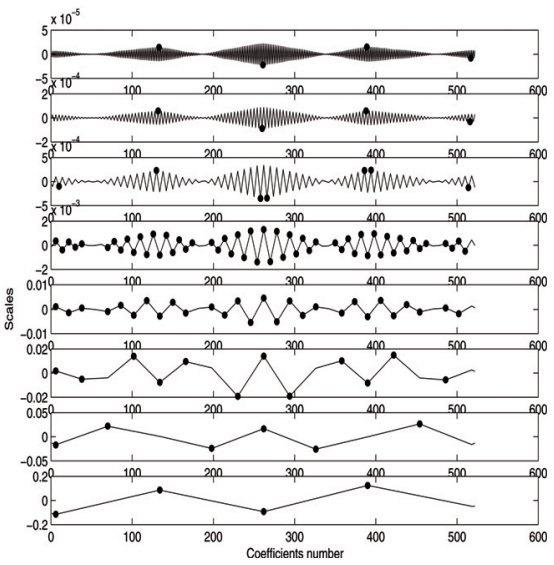

(d)

Fig. 7. Some results for the synthetic light curve given by Eq. (17): a) and c) show the noisy data with $S N R=30$ and $1 \mathrm{~dB}$, respectively, the filtered curve, and the results got by the algorithm; b) and d) show the upsampled wavelet coefficients $d_{j}$ for the noisy signals at scales $j=1, \ldots 8$. Circles mark the local modulus maxima wavelet coefficient.

precise results for $S N R$ values above $20 \mathrm{~dB}$. Taking into account that the sampling rate is $0.84 \mathrm{~s}$, the estimated error reaches up to 15 samples when the noise level in the signal is high (low $S N R$ ) and goes down to at most 1 sample when the $S N R$ is above $26 \mathrm{~dB}$.

These results allow us to compute the accuracy of the method when it is applied to our experimental data from the PHEMU campaign. An estimate of the SNR level of the data gives a value of $27.6 \mathrm{~dB}$, which is above the lower limit of $20 \mathrm{~dB}$ required to obtain an accurate estimation.

\subsection{Processing of the light curve from J1 ECL J2}

We now apply the provided strategy to a set of experimental data. The considered light curve corresponds to the eclipse of Europe by Io, as observed from the Royal Observatory at San Fernando, Cádiz (Spain) on the 7th July 1997. Recorded data were stored in a file where the number of the record was given from left to right, along with the time of the beginning of each record in hours, minutes, and seconds, the recorded voltage, and the time when the record ends, as shown in Table 2. The first ten records and the last ten ones match the sky background.
In order to perform the wavelet decomposition, it was necessary to increase the sample to reach a power of 2 . New data were added specularly, inserting each half on both sides of the signal. This does not influence our results significantly since first and last data are related to the sky background, when the event had not begun or had already finished.

The filtering of the data using the coherent structures algorithm produces the denoised light curve plotted in Fig. 9. In this case the best wavelet basis for the denoising is the Daubechies 20.

We ran the maxima curves algorithm with several wavelet basis. The results lead us to conclude that the bases which detect the light curves better are the Coiflet of two vanishing moments and the Biorthogonal of two vanishing moments. The upsampled wavelet coefficients associated to these wavelet bases are shown in Figs. 8a and b. The times estimated by the algorithm are marked on the filtered data in Fig. 9 and summarized in Table 3.

In order to estimate the value of the $S N R$ of our signal, we assume that the pre-event and post-event data provide a good estimation for the entire noise data, and we obtain a value of $S N R=27.6 \mathrm{~dB}$. According to the results for the case of the 
Table 1. Outline of the results obtained for a synthetic light curve, with SNR ranging from $1 \mathrm{~dB}$ to $40 \mathrm{~dB}$, using a Biorthogonal basis.

\begin{tabular}{|c|c|c|c|c|c|c|c|c|c|}
\hline \multirow[b]{2}{*}{$S N R$ level } & \multicolumn{3}{|c|}{$t_{\mathrm{ini}}^{s}=-98.88$} & \multicolumn{3}{|c|}{$t_{\min }^{s}=0.24$} & \multicolumn{3}{|c|}{$t_{\mathrm{end}}^{s}=98.52$} \\
\hline & $t_{\text {ini }}$ & Error (s) & No. of samples & $t_{\min }$ & Error (s) & No. of samples & $t_{\text {end }}$ & Error (s) & No. of samples \\
\hline 1 & -111.48 & +12.6 & 15 & -3.96 & +4.2 & 5 & 110.28 & -11.76 & 14 \\
\hline 2 & -111.48 & +12.6 & 15 & -3.96 & +4.2 & 5 & 110.28 & -11.76 & 14 \\
\hline 3 & -111.48 & +12.6 & 15 & -3.96 & +4.2 & 5 & 110.28 & -11.76 & 14 \\
\hline 4 & -111.48 & +12.6 & 15 & -3.96 & +4.2 & 5 & 110.28 & -11.76 & 14 \\
\hline 5 & -111.48 & +12.6 & 15 & -3.96 & +4.2 & 5 & 110.28 & -11.76 & 14 \\
\hline 6 & -111.48 & +12.6 & 15 & -3.96 & +4.2 & 5 & 111.96 & -13.44 & 16 \\
\hline 7 & -111.48 & +12.6 & 15 & -3.96 & +4.2 & 5 & 111.96 & -13.44 & 16 \\
\hline 8 & -111.48 & +12.6 & 15 & -3.96 & +4.2 & 5 & 111.96 & -13.44 & 16 \\
\hline 9 & -111.48 & +12.6 & 15 & -3.96 & +4.2 & 5 & 111.96 & -13.44 & 16 \\
\hline 10 & -111.48 & +12.6 & 15 & -3.96 & +4.2 & 5 & 111.96 & -13.44 & 16 \\
\hline 14 & -111.48 & +12.6 & 15 & 0.24 & 0 & 0 & 111.96 & -13.44 & 16 \\
\hline 16 & -103.08 & +4.2 & 5 & 0.24 & 0 & 0 & 111.96 & -13.44 & 16 \\
\hline 18 & -103.08 & +4.2 & 5 & 0.24 & 0 & 0 & 111.96 & -13.44 & 16 \\
\hline 20 & -103.08 & +4.2 & 5 & 0.24 & 0 & 0 & 111.96 & -13.44 & 16 \\
\hline 22 & -98.04 & -0.84 & 1 & 0.24 & 0 & 0 & 103.56 & -5.04 & 6 \\
\hline 24 & -98.04 & -0.84 & 1 & 0.24 & 0 & 0 & 103.56 & -5.04 & 6 \\
\hline 26 & -98.04 & -0.84 & 1 & 0.24 & 0 & 0 & 98.52 & 0 & 0 \\
\hline 28 & -98.04 & -0.84 & 1 & 0.24 & 0 & 0 & 98.52 & 0 & 0 \\
\hline 30 & -98.04 & -0.84 & 1 & 0.24 & 0 & 0 & 98.52 & 0 & 0 \\
\hline 32 & -98.04 & -0.84 & 1 & 0.24 & 0 & 0 & 98.52 & 0 & 0 \\
\hline 34 & -98.04 & -0.84 & 1 & 0.24 & 0 & 0 & 98.52 & 0 & 0 \\
\hline 36 & -98.04 & -0.84 & 1 & 0.24 & 0 & 0 & 98.52 & 0 & 0 \\
\hline 38 & -98.04 & -0.84 & 1 & 0.24 & 0 & 0 & 98.52 & 0 & 0 \\
\hline 40 & -98.04 & -0.84 & 1 & 0.24 & 0 & 0 & 98.52 & 0 & 0 \\
\hline
\end{tabular}

Table 2. File containing the original data.

\begin{tabular}{ccccccc}
\hline \hline Record & Hour & $\min$ & $\mathrm{s}$ & Voltage & $\min$ & $\mathrm{s}$ \\
\hline 1 & 2 & 8 & 20.82 & 0.449219 & 8 & 21.10 \\
2 & 2 & 8 & 21.59 & 0.463867 & 8 & 21.81 \\
3 & 2 & 8 & 22.36 & 0.478516 & 8 & 22.58 \\
4 & 2 & 8 & 23.13 & 0.463867 & 8 & 23.35 \\
5 & 2 & 8 & 23.90 & 0.468750 & 8 & 24.17 \\
6 & 2 & 8 & 24.67 & 0.473633 & 8 & 24.89 \\
7 & 2 & 8 & 25.44 & 0.478516 & 8 & 25.66 \\
8 & 2 & 8 & 26.37 & 0.463867 & 8 & 26.70 \\
9 & 2 & 8 & 27.30 & 0.434570 & 8 & 27.52 \\
10 & 2 & 8 & 28.02 & 0.468750 & 8 & 28.29 \\
. &. &. &. &. &. &. \\
\hline
\end{tabular}

synthetic light curve, we can conclude that the associated error is $0.84 \mathrm{~s}$, i.e., the sampling size.

\section{Discussion}

The remarkable point of this method is the denoising strategy and the effectiveness of the designed algorithm in detecting the maxima curves and, therefore, the three searched times. Firstly, from what appeared to be chaotic data, the filtering method provides a consistent light curve that fits the theoretical one. It should also be definitely noticed that no information about the expected curve is used at any point in the denoising process.

Secondly, when running the designed algorithm with the chosen wavelets (Coiflet of two vanishing moments and Biorthogonal of two vanishing moments), three maxima curves are detected, converging to the abscissas that match two points of maximum curvature and the minimum in the light curve, which are the instants of time we are looking for. We do emphasize the fact that the algorithm has no previous information, that is, the algorithm detects three special points, without any a priori knowledge or characterization of them.

The times we get with both wavelet functions are exactly the same, except for the time of minimum flux, which differs from one to the other by one sample. This minimum difference shows the mathematical accuracy of the proposed method, since it falls in the range of the predicted error.

Finally, it must be noticed that the obtained results are not exactly the times of the beginning, end, and minimum light flux of the phenomenon, since we did not carry out any correction to the data. In this way, albedo markings, limb-darkening, and illumination geometry must be taken into account as soon 


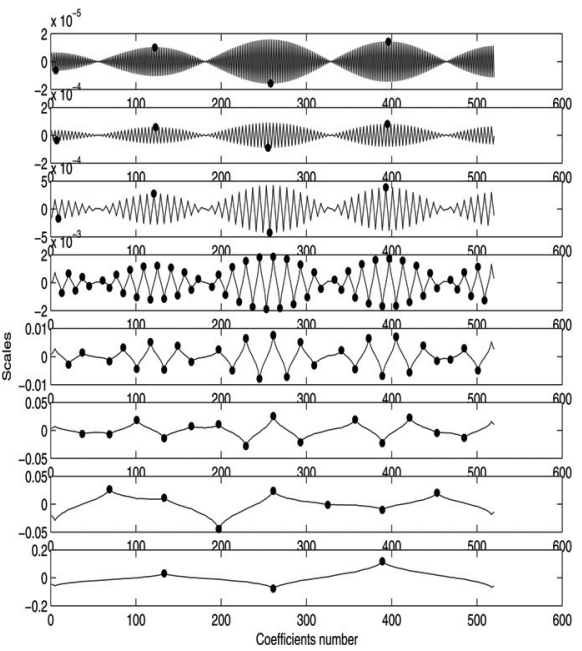

(a)

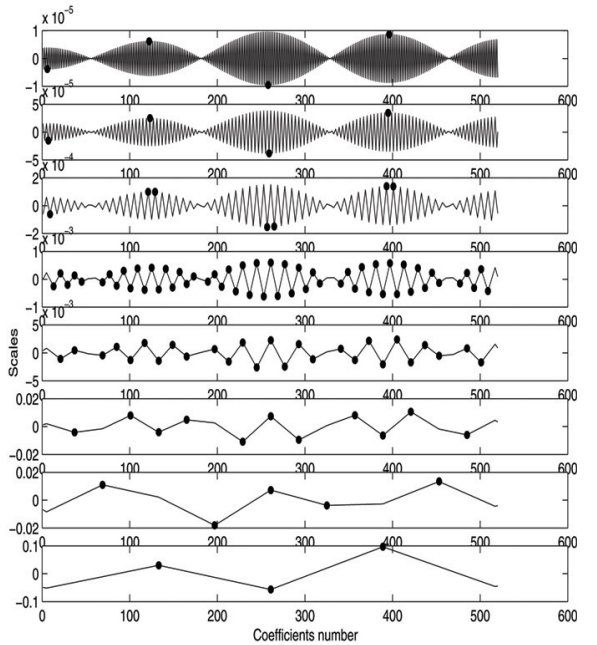

(b)

Fig. 8. Upsampled wavelet coefficients $d_{j}, j=1 \ldots, 8$ of the synthetic light curve using a) a Coiflet basis with two vanishing moments and b) a Biorthogonal basis with two vanishing moments. The marked points show the modulus maxima wavelet coefficients.

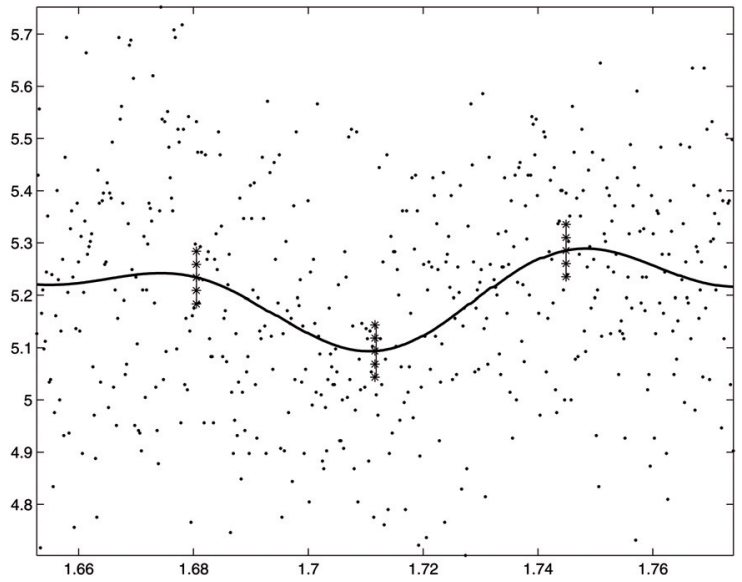

(a)

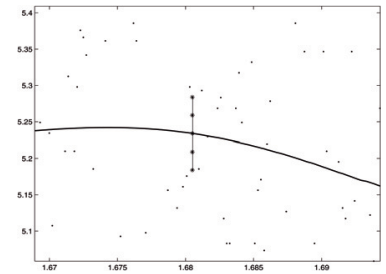

(b)

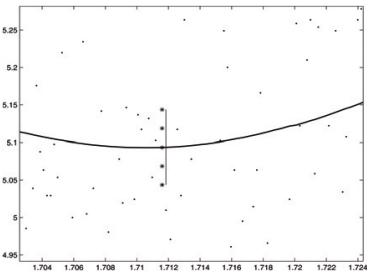

(c)

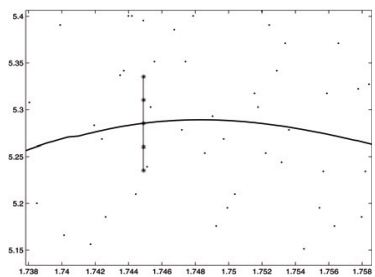

(d)

Fig. 9. a) Data filtered by means of the coherent structure procedure. Times obtained by using a Coiflet basis are marked with a solid line (-); times got by means of a Biorthogonal basis are marked with an asterisk (*). b)-d) Zooming onto these three steps.

Table 3. Estimated times $t_{\mathrm{ini}}, t_{\min }, t_{\mathrm{end}}$ for the eclipse of Europe by the satellite Io. They were obtained by applying the maxima curves algorithm with a Coiflet wavelet basis and a Biorthogonal wavelet basis, both with two vanishing moments. The initial and end event times coincide with both wavelet functions, and only the moment of minimun flux differs.

\begin{tabular}{cccc}
\hline \hline Wavelet basis & $t_{\text {ini }}$ & $t_{\min }$ & $t_{\text {end }}$ \\
\hline Coiflet 2 vanishing moments & $1: 40: 49.91 \mathrm{UT}$ & $1: 42: 41.83 \mathrm{UT}$ & $1: 44: 41.57 \mathrm{UT}$ \\
Biorthogonal 2 vanishing moments & $1: 40: 49.91 \mathrm{UT}$ & $1: 42: 42.62 \mathrm{UT}$ & $1: 44: 41.57 \mathrm{UT}$ \\
\hline
\end{tabular}


as we are interested in giving a physical meaning to the obtained times. In this paper, our purpose has only been to show the possibility of studying astronomical data using the wavelet techniques.

A detailed study of the rest of the phenomena in Fig. 4 introducing those corrections will be the subject of a future work.

Acknowledgements. Part of this work was supported by the group Geodesia y Geofísica- RNM314, belonging to the Junta de Andalucía, Spain. We would also like to thank the Real Instituto y Observatorio de la Armada in San Fernando (Cádiz) for supplying the astronomical data we analyzed.

\section{References}

Arlot, J. E. 1987, Ann. Phys. fr., 12, 7

Arlot, J. E., Thuillot, W., Barroso Jr., J., et al. 1992, A\&A, 92, 1

Arlot, J. E. 1997, A\&A, 314, 312
Arlot, J. E., Berthier, J., Colas, F., et al. 1997, Mutual Events of the Galilean Satellites: the PHEMU97 Campaign. In the 4th Workshop on Positional Astronomy and Celestial Mechanics Donoho, D., \& Jonhstone, I. 1994, Biometrika, 81, 425

Gil Chica, F. J. 1996, Teoría de eclipses, ocultaciones y tránsitos, Universidad de Alicante

Hwang, W. L., \& Mallat, S. 1991, Singularity Detection and Processing with Wavelets, Technical Report (New York University)

Mallat, S. 1999, A Wavelet Tour of Signal Processing (London: Academic Press)

Pazos, A., González, M. J., \& Alguacil, G. 2003, Journal of Seismology 7, 413

Sánchez-Francisco, M., Salazar, A., Coll y Ramón, V., et al. 1998, La Campaña Phemu'97, Boletín ROA No. 2/98 Real Instituto y Observatorio de la Armada en San Fernando

Vetterli, M., \& Kovackevic, J. 1995, Wavelets and Subband Coding (New Jersey: Pearson Education) 